\title{
Calidad escolar: un nuevo ethos escolar
}

\section{School quality: a new ethos}

\author{
Laura Ramírez Saborio \\ Directora de la Escuela de Educación \\ Decana de Ciencias Sociales, Universidad Latina de Costa Rica \\ San José, Costa Rica \\ laura.ramirez@ulatina.cr
}

Recibido: 27-II-2011 • Aceptado: 02-IV-2012 • Corregido: 16-XI-2012

\begin{abstract}
Resumen: Actualmente ha cobrado vigencia y preocupación el tema de la calidad escolar, por cuanto la educación se considera como la herramienta más poderosa de transformación en una sociedad. Se necesitan herramientas y referentes teóricos para construir el concepto de calidad y el ethos, $y$, para traducirlos en estrategias prácticas y realidades en nuestro sistema educativo. Se toman como base varias investigaciones para analizar la temática y se plantean ideas concretas que podrían permitir la implementación de políticas.
\end{abstract}

Palabras clave: Educación, calidad.

Abstract: Education is considered the most powerful tool in attaining the transformation of society. As a result, school quality has become an issue in today's society. For this transformation to happen, tools and theoretical references are needed. They would help construct this concept of quality and translate it into practical strategies and realities applicable to our educational system. In order to analyze this topic, several research studies are used and concrete ideas that might help implement the new policies are proposed.

Keywords: Education, quality.

\section{Propósito}

Este documento pretende ofrecer una aproximación teórica a los elementos que debe contemplar el concepto de calidad escolar. Para realizar este trabajo, se han tomado como base diversas investigaciones y propuestas que han abordado dicho tema. Cada una de ellas se aproxima el tema de la "calidad escolar" con distinta óptica, las cuales no necesariamente son excluyentes.

El objetivo del artículo es, elaborar en un primer intento, lo que implica la calidad para nuestros sistemas educativos, tomando como base textos, estudios y artículos varios.

Los apartados obedecen a una propuesta de ideas o pistas que podrían considerarse, en la construcción del concepto de educación de calidad. 


\section{Dos temas para reflexionar y hacer en calidad educativa}

De los estudios considerados, se rescatan dos ideas puntuales que permiten tener un norte claro sobre lo que se puede hacer para mejorar la calidad educativa.

\subsection{Una apuesta a favor de los- formadores}

Educación parvularia o preescolar (primera infancia): profesorado y madres de familia.

Los datos sobre la educación parvularia apuntan a dos acciones: reflexionar e inmediatamente "hacer". El nivel preescolar ya no puede ser asistencial ni de baja calidad en los sectores pobres. La "educación preescolar de calidad contribuye a desarrollar las habilidades académicas" (Eyzaguirre y Le Foulon, 2001, p. 91). Este estudio y el de Arancibia (1998) confirman la importancia de los antecedentes preescolares como variables que afectan el rendimiento.

Entre los elementos señalados -en los estudios previamente mencionados - se encuentra que la mayoría de "las educadoras de párvulos tienen un escaso dominio de la estimulación cognitiva y desaprovechan el tiempo con esperas ociosas y actividades rutinarias que no aportan al desarrollo intelectual y a la madurez de los alumnos" (Eyzarguirre y Le Foulon, 2001, p. 101). Así, entre las variables responsables relacionadas con la baja calidad en los programas de educación preescolar está el quehacer del profesorado y de las instituciones. En el estudio citado se señalan dos puntos:

- No existe diferencia en el nivel cognitivo si los niños y niñas asisten o no a un parvulario (incluso a veces asistir a uno disminuye el desarrollo intelectual).

- $\quad$ En este sentido, atañe a las instituciones educativas y muy especialmente a 
los profesores el tipo de educación que están dando en los establecimientos.

De esta manera, es relevante y merecedor hacer un estudio para saber qué dicen conocer y qué hacen los docentes en distintas áreas y contrastarlo con la realidad. En Costa Rica, por ejemplo, la mayoría de las docentes en el área del lenguaje no conocen bien el programa de estudios y la mayoría de las veces no saben para qué desarrollan algunas estrategias de enseñanza de la lengua materna, aún cuando algunas son positivas para el aprendizaje del estudiantado en el nivel preescolar. (Ramírez, 1999).

Por lo tanto, es necesario:

1. Investigar lo que el cuerpo docente conoce sobre los programas de estudio que imparten (los contenidos).

2. Analizar los programas que ofrecen las diferentes universidades en la formación de educadores así como los cursos de capacitación permanente.

3. Hacer un análisis crítico y exhaustivo de los criterios de selección del personal docente en los diferentes niveles del sistema educativo. Sería deseable incluir no solo el sector público, sino también el sector privado.

Las investigaciones sobre este tema en distintas regiones del mundo donde existen distintas calidades en la formación docente (Ross y Postlewhite, 1992, en África, América Latina y Asia) sugieren que -con algunas excepciones- la formación de los docentes está asociada con los rendimientos de los alumnos (Laboratorio Latinoamericano de la Evaluación de la calidad de la educación -LLECE en adelante- 2001, p.94).

Asimismo, en el estudio de Arancibia (1998), una de las variables socioculturales relevantes es la educación de los padres y el nivel socioeconómico (NSE) de la familia. La autora señala como fundamental el rol socializador de las madres especialmente en el nivel socioeconómico bajo, "la madre concentra el cuidado y la educación de los hijos en las primeras etapas del desarrollo" (Arancibia, 1998, p. 179).

Luego de este recorrido, se debe:

- Retomar y analizar la formación que están recibiendo las docentes para el nivel inicial. Esto incluye un análisis curricular de lo que hacen las universidades para estas profesionales.

- Replantear e incorporar el tema de la madre trabajadora, como una variable decisiva en la formación de sus hijos.

Es decir, el nivel preescolar determina en gran medida el rendimiento posterior de los estudiantes, por ello a este nivel se le debe prestar especial importancia.

Siteal (2009, citado en el Tercer Informe del Estado de la Educación, 2011) -donde se dedica un exhaustivo capítulo a explicar la importancia de la educación preescolar y su importancia para el desarrollo de la niñez y de la sociedad -, indica cómo los primeros años de la niñez son fundamentales para el desarrollo de las conexiones neuronales y recomienda la estimulación de este desarrollo, a partir de ambientes ricos desafiantes. Siteal indica que si existe esta estimulación hay mayor obtención de éxitos en la trayectoria escolar evitando fracasos y repeticiones:

las razones pedagógicas que justifican la educación temprana en instituciones educativas se vinculan con la importancia de una acción sistemática, adaptada a las características y necesidades de la edad, lo que contribuye en gran medida al mejor desarrollo de la personalidad de cada niño y a su posterior desempeño escolar" (p. 67).

Eyzaguirre y Le Foulon no pueden ser más claras cuando afirman:

...La educación preescolar no se puede justificar sólo por su contribución al desarrollo intelectual y emocional de 
los niños, sino que también porque cumple funciones de guardería. Los jardines infantiles permiten el trabajo de las madres, el cual es un objetivo deseable ya que aumenta el ingreso familiar. (Eyzaguirre y Le Foulon, 2001, p. 104)

\section{2 ¿Qué nos enseña Cuba?}

Los niños cubanos de la escuela general básica obtienen resultados que se escapan de la mediana regional -constituida en este estudio por Argentina, Bolivia, Brasil, Chile, Colombia, Honduras, México, Paraguay, República Dominicana y Venezuela - evaluados en áreas como el lenguaje y matemática (LLECE, 2000). Cuba se distingue como el único país con puntajes muy superiores a la mediana y significativamente mayores que los de otros países (Eyzaguirre y Le Foulon, 2001, p. 145).

Este país nos enseña que invertir en los profesores es uno de los pilares para que la escuela cumpla una de sus funciones: que los niños aprendan. Pero, ¿cómo lo hacen?

En Cuba, el único tipo de escuela existente es la escuela pública. Ya esto es una enseñanza para el resto de los sistemas educativos en Latinoamérica, cuando a la luz de datos como los que se brindan en el TIMSS y el IALS (citados en Eyzaguirre y Le Foulon, 2001), Cuba tiene rendimientos incluso por encima de la media.

Froemel (2002) señala varios puntos que nos aclaran algunas de las virtudes del sistema cubano:

- $\quad$ En Cuba se hace en educación todo lo que los libros dicen que hay que hacer.

- El 98 \% de la población preescolar tiene estimulación temprana (en los jardines infantiles, las madres encargadas de este proceso).

- $\quad$ Sobre el profesorado:

- Están sometidos a un control social. Es decir, rinden cuentas sobre su trabajo.
- Tienen un año sabático cada cincos años.

- Si fracasan en su labor pueden perfeccionarse, si vuelven a fallar dejan de ser profesores.

Ahora bien, lo anterior es lo que debemos de considerar, porque aún con los altos rendimientos que tienen los estudiantes, tampoco es un secreto la contradicción "entre una educación capaz de dar alas a los niños y jóvenes y una sociedad que las corta, al impedir la crítica y el debate" (Ednir, 2002, p. 76).

El compromiso vocacional del docente lo conduce a no solamente querer realizar una buena labor profesional, sino que también se entrelaza con las expectativas que tiene de sus alumnos. No se trata por tanto de lograr un buen resultado, sino de afectar positiva e integralmente a los niños/as. El docente de estas escuelas es innovador y abierto a las nuevas ideas, se encuentra constantemente en la búsqueda de probar nuevos métodos para lograr el objetivo de educación integral para los alumno/as. (LLECE, 2002, p.14)

Para finalizar, se retoma una idea de Stigler y Hiebert en el que concluyen que "la llave para mejorar la calidad de la educación está en que los profesores dispongan de mecanismos efectivos para mejorar continuamente su docencia ${ }^{1}$, y no así en los gastos en infraestructura, equipamiento o programas de educación especial” (Stigler y Hiebert, 2002, p. 57).

\section{Calidad escolar: un concepto en construcción}

Arancibia y Cassasus (1996) señalan que sobre la calidad siempre se va a emitir un juicio. Los juicios se construyen socialmente. El aporte del texto es que señala que "calidad" está ligada al tema de la equidad. Esto no es menor pues pone de antemano un tema de justicia social.

Para hablar de la calidad de la educación habría que considerar los siguientes puntos: 
- Rendimiento académico del estudiantado.

- Formación del equipo de profesores.

- $\quad$ Nivel socioeconómico de los padres y madres de familia o encargados

- $\quad$ Condiciones socioculturales de la niñez.

- Elaboración de políticas públicas con compromiso social y criterios de expertos.

Los anteriores puntos se reúnen integradamente como reflexión en los siguientes apartados.

\subsection{Responsabilidad ética de la educación}

El concepto de calidad no se puede restringir a los resultados de las pruebas estandarizadas nacionales o internacionales.

Resulta muy difícil distinguir entre calidad de la educación e igualdad de oportunidades (OCDE, 1991, citado en Arancibia y Casassus, 1996). Lo que habría que revisar son tanto las oportunidades como las CONDICIONES en que se da la educación para los sujetos menos aventajados socioeconómicamente hablando.

De acuerdo con Arancibia y Casassus:

...La masificación plantea problemas pedagógicos completamente distintos. La preocupación pública asume la calidad de la educación, la convierte en objeto de política pública... Hay un acceso diferenciado a la calidad que ofrece el sistema. Las maneras para acceder a esa calidad no tienen por qué ser las mismas para todos... En la actualidad, la educación con estándares comunes puede ser considerada un bien público (sic), mientras que aquélla con demandas particulares puede ser considerada un bien privado. (Arancibia y Casassus, 1996, p. 16)

Siempre que haya educación de calidad, en cualquier nivel, van a existir dos beneficiarios: el individuo y la sociedad.

\section{Objetivos de la escuela} señalan: ...dependiendo de la unidad de análisis los objetivos y desempeño de las escuelas son distintos: una unidad de análisis puede operar desde la óptica del Estado (desarrollo de los individuos, ciudadanía educada, otros pueden ser desde la escuela misma); otra unidad de análisis sería los objetivos de la escuela (las preocupaciones por el desarrollo emocional, cognitivo y psicológico de los niños). (Arancibia y Casassus, 1996, p. 24)

Aquí nos encontramos una vez más con disyuntivas de carácter político, puesto que no existe entre los especialistas un acuerdo acerca de un marco de referencia único a partir del cual deducir indicadores. Vale decir sobre esto que no tendría por qué haber contradicción: los estándares desde el nivel macro o micro son distintos pero no excluyentes. Desde el nivel macro (por ejemplo el Estado), se plantean objetivos generales que deben operacionalizarse en el nivel micro (con cada alumno, con cada tarea y programa de estudios que se desarrolla pero sin que se pierda el objetivo o norte que los guía: el Estado). Otra cosa sería si la escuela no está de acuerdo con lo que pide o demanda el Estado. Por ejemplo que los profesores, alumnos o padres de familia no estén de acuerdo con lo que el Estado establece.

\subsection{Capital social: una nueva mirada a la educación}

Cualquier óptica de efectividad y calidad escolar debe considerar el concepto de "capital social". Algunas de las definiciones de la importancia del capital y desarrollo humano están dadas por el Banco Mundial. Se ubica desde la perspectiva económica, pero especialmente de lo que se llama "Desarrollo humano" en el Programa de Naciones Unidas para el Desarrollo (PNUD). Este estaría entendido de la siguiente manera:

El desarrollo humano sostenible (DHS) es concebido [...] como un proceso continuo e integral que reúne componentes 
y dimensiones del desarrollo de las sociedades y las personas, en los que resulta central la generación de capacidades y oportunidades de, por y para la gente, con las que la equidad se acreciente para las actuales y futuras generaciones. (Estado de la Nación, 2000, p. 28)

El otro concepto que debería incorporarse al de la calidad de la educación es el del capital social, que según Bertucci (2002) "se refiere a las instituciones, relaciones y normas que conforman la calidad y cantidad de las interacciones sociales de una sociedad (...) No es sólo la suma de las instituciones que configuran una sociedad, sino asimismo la materia que las mantiene juntas" (p.1). De acuerdo con el Banco Mundial (2002), "numerosos estudios demuestran que la cohesión social es un factor crítico para que las sociedades prosperen económicamente y para que el desarrollo sea sostenible".

Otra definición del Banco Mundial explica que el capital social tiene implicaciones importantes tanto para la teoría como para la práctica y la política del desarrollo de las sociedades. Las "recetas" convencionales para mejorar las perspectivas económicas de las comunidades y naciones incluyen mejorar la educación y los servicios de salud, entre otros. Coleman y Hoffer (1987), Braatz y Putnam (1996) y Francis et al. (1998) (citados en Banco Mundial, 2002) señalan sobre el capital social y la educación:

Las escuelas son más efectivas cuando los padres y los ciudadanos locales se involucran en sus actividades. Los maestros están más comprometidos, los estudiantes alcanzan mejores resultados en los exámenes y se usan mejor las instalaciones de las escuelas en aquellas comunidades en las cuales los padres y ciudadanos se interesan en el bienestar educativo de los niños.

\subsection{Un nuevo ethos escolar}

Es muy valioso que autores como Bellei (2001) incorporen en la discusión sobre calidad el tema de la "cultura escolar" y la "tradición docente", pues como su mismo texto lo señala, estos elementos son los más difíciles de modificar.

Dentro del concepto de calidad de educación se debería de hablar y reconceptualizar una nueva "forma de ser" o ethos de la escuela. En este ethos se incluirían las reflexiones realizadas líneas atrás. En síntesis, se trata de darle una cara y una personificación distinta a la escuela, que aspire de nuevo a ser un instrumento de movilidad social de las personas que acceden al sistema escolar, pero con exigencia en los distintos niveles del propio sistema.

Por ejemplo, a partir de los distintos resultados vemos que hay varios factores que afectan la calidad educativa. Una óptica de abordar ese ethos es apuntando a aquello que parece ser más débil o deficitario. Una muestra se refiere a las formas de interacción profesor-alumno.

Estas formas son más activas en la actualidad (Bellei, 2001) aunque, según los datos de Eyzaguirre y Le Foulon (2001), no han incidido - hasta el momento - en el mejoramiento de habilidades de alto orden. La respuesta que Bellei daría a Eyzaguirre sería que:

Los docentes chilenos realizan un tipo de prácticas de enseñanza que, sin apartarse radicalmente de la tendencia general, muestra algunos desfases susceptibles de ser mejorados para determinar [...el] tipo de métodos de enseñanza empleados están o no apuntando a lograr mayores y mejores aprendizajes, es imprescindible acceder a un tipo de información no estandarizada, obtenida en contexto reales de trabajo escolar. (Bellei, 2001, p.30)

Hay claridad en cuanto a los cambios que deberían operar en relación con el rol del profesor (Eyzaguirre y Le Foulon, 2001; Arancibia, 1993; Bellei, 2001; Froemel, 2002; entre otros). Igualmente, hay que recordar que Fullan (2000) explica que para que haya cambio son necesarios varios actores.

Esto debería incluir una evaluación verdadera del sistema desde el nivel micro hasta el macro. Tal como lo señala 
Carbajosa (2007) - en una nota periodística sobre el modelo educativo finlandés -, los educadores deciden qué enseñan, cómo lo enseñan, a quién y a qué ritmo. Tienen tanta libertad como preparación. Al finalizar el año, profesores, estudiantes y padres "evaluarán los objetivos que los propios alumnos se fijaron al comenzar el curso y se pondrán nota”. En este país modelo en educación, la evaluación continua, es decir, el aprendizaje y la actitud del joven durante todos los días del año. Este mismo autor describe lo que dice Meri -uno de los profesores de la Facultad de Pedagogía de Helsinki, y se refiere a la necesidad de evaluarse de forma permanente -. "El ser humano tiene que ser capaz de fijarse sus propios objetivos, y después, ser capaz de evaluarse. Hay que hacerles responsables de su propia vida desde el principio, que aprendan a no delegar en la sociedad la responsabilidad de sus actos". Meri (sf) cree además que es fundamental establecer una relación de iguales entre profesores y alumnos. "El maestro no tiene que saber mucho. Tiene que saber escuchar. A veces, es más importante escuchar al alumno y compartir sus conocimientos. En Finlandia, los profesores y los alumnos se respetan mucho, pero no desde la jerarquía, sino desde la igualdad" (Meri, s.f., citado por Carbajosa, 2007).

\section{Algunas dimensiones de este nuevo ethos}

Arancibia y Cassasus (1996) señalan seis dimensiones de la calidad, con las cuales coincidimos. Estas son:

1. Objetivos y las metas de la escuela o del sistema. El producto o tipo de servicio que busca entregar la escuela.

2. Estilos de planificación, gestión y evaluación y su relación con las expectativas de resultados. La cultura de la calidad, el liderazgo del director en los compromisos y las motivaciones derivadas de la participación y en la autonomía de acción.

3. Facilidades de acceso al aprendizaje.

4. Docentes y su actividad.

5. Ambiente emocional.

6. Área de vínculos con los padres y apoderados de los niños. Este es uno de los factores que más influye en el rendimiento de los alumnos.

Hay que ser enfáticos. Estas dimensiones son la guía para establecer los estándares de la calidad de educación requerida. La complejidad de ópticas que operan hace ver el tema de la calidad como un ideal. Sin embargo, con el planteamiento de políticas claras se puede llegar a concretar en el tiempo los efectos deseados.

Es momento de diseñar parámetros y estándares concretos de calidad. Evidentemente la discusión está en su pleno apogeo y la construcción de distintos discursos ya está en la mesa de discusión, ópticas distintas igualmente necesarias. Debemos crear nuevas formas de pensar la educación de calidad.

Quizá el punto focal sea el tratamiento que se le debe dar a la formación del profesorado pues ellos son los que marcan la diferencia. Tal como se señala en el Tercer Informe del Estado de la Educación:

En materia de formación y desarrollo profesional docente, los estudios más recientes y las mejores prácticas inter $\neg$ nacionales señalan que la calidad de los maestros y profesores es el factor que más influye en la calidad de la educación. Para avanzar en este ámbito es importante mejorar los procesos de selección de los estudiantes en las carreras de Educación y, en particular, lograr la acreditación de calidad de los programas universitarios de formación docente. Una necesidad urgente es que el MEP cuente con un perfil del educador que desea contratar, y que pida como requisito la acreditación de la carrera universitaria de la que provienen las y los interesados. (Estado de la Educación, 2011, p. 39)

Eso sí, el tema de la acreditación es tema profundo para otro estudio o artículo. En lo que no hay duda es que es necesaria 
la participación activa de varios actores en la construcción de ese concepto de calidad. Arguedas y Bonilla (2008) señalan que si la comunidad completa es partícipe de los procesos educativos y de desarrollo que se dan en ella, habrá mayores logros en cuanto a la calidad y equidad, por medio de la vinculación en proyectos comunes para el beneficio de toda la población.

\section{Temas para la discusión}

El concepto de calidad está en permanente evolución. Efectivamente hay parámetros claros que permiten definir estándares para saber si un sistema educativo tiene o no calidad escolar: en el tema de la calidad están relacionados aspectos tales como la formación del cuerpo docente, las condiciones socioculturales de la niñez de 0 a 6 años, la calidad de la educación inicial (o parvularia) que reciben los niños y niñas, el ethos escolar y la incorporación del concepto del capital social ligado al de calidad. Hasta el momento estos son los que para nosotros definirían en gran medida la calidad educativa. Sin embargo, como no es una postura absoluta, se plantean los siguientes puntos para la discusión:

- Es necesario recalcar la idea de que la educación no es neutral. Esto significa que cualquier decisión en políticas públicas afectará directamente -a estudiantes y profesores - e indirectamente - a padres de familia y la sociedad en general - los intereses y necesidades de los distintos actores.

- El saber qué se está haciendo y qué se debe modificar en la formación de profesores y su evaluación es urgente, tal como lo indica el último Informe del Estado de la Educación. Y como dice Margery, "pocos profesores son conscientes de que nuestro actual sistema de enseñanza responde a la visión mecanicista del mundo (su ideal paradigmático es la cadena de montaje)" (Margery, 2010, p. 72). La calidad escolar tiene que ver con la construcción de un nuevo ethos escolar. ¿Qué somos ahora, pero sobre todo ¿qué queremos ser?, ¿cómo es ese estudiante ciudadano que se aspira tener?

- $\quad$ Es evidente la necesidad de que haya un planteamiento que remueva, que genere cuestionamientos y aportes. Hay con dos aspectos puntuales que señala Espínola (1998): debe haber mayor designación de recursos a las escuelas públicas y se deben generar estrategias de focalización como base para el diseño de políticas públicas.

- La educación para todos no es una frase hecha o eslogan, el tema de educación de calidad es un derecho. Entonces, educación para "los más pobres" tiene un aire de discriminación y de medida preventiva. Es menester de quienes laboramos en el área educativa generar esa educación de calidad no importa la población con la que trabajemos. Arancibia (1993) señala que es de vital importancia el papel que debería replantearse la escuela en la formación y no en la instrucción, punto con el que coincido. Deben por otro lado aprovecharse todas aquellas situaciones relacionadas con el área afectivo-social pues podrían potenciarse mejores relaciones humanas (aspecto reclamado por los estudiantes) y mejores rendimientos académicos.

Se debe retomar la voz de los estudiantes. Muchas de las opiniones de los jóvenes (Arancibia, 1993) se relacionan con el "ideal", sobre lo que quisieran en educación. No es que ellos deban indicar "hacia donde se va” en sentido estricto, pero su voz debe ser considerada. "La juventud se caracteriza por el sentimiento de tener una meta, quizá no precisada, pero sí sentida al menos como futuro afortunado de aquello que 
se está viviendo" (Guissani, 2006, p. 56); o como lo hace la filosofía de Reggio Emilia, reflexionando sobre la cultura infantil y la necesidad de volver la mirada atenta a ellos. Así dice Hoyuelos, "la escuela tiene que tener la característica, sobre todo, de escuchar cómo es la cultura de la infancia, que muchas veces no se corresponde con la idea que el adulto tiene sobre la infancia. Recogiendo las ideas de los niños y las niñas, el adulto puede transformar su propia cultura a partir de los valores o del punto de vista que tienen los niños y niñas sobre la sociedad y la cultura" (Hoyuelos, s.f., p. 3)

- Muchas de las políticas educativas y sociales de los gobiernos deben ir encaminadas al mejoramiento de los factores: nivel sociocultural, formación de profesores y madres de familia y apoderados en general, objetivos de calidad en educación. Ardua tarea, posiblemente sin muchas respuestas al corto plazo, pero de obligación en una sociedad que dice llamarse democrática. Como lo dice Guissani (2006), "el gran problema de la sociedad es ante todo educar a los jóvenes (lo contrario de lo que sucede ahora)" (p. 15).

- $\quad$ Apoyando la idea de Arancibia de que "la dinámica del proceso educativo está determinada por una multiplicidad de factores que interactúan, y cuyo efecto en el rendimiento de los alumnos depende de aspectos culturales, económicos y demográficos" (1998, p. 197), se remite a la idea, de que para que se genere cambio es necesario una visión sistémica de la educación.

- Es necesario seguir explorando este "ethos escolar". Esa idea que va unida a las metas de la institución, identidad institucional y el proyecto de escuela en general.

Se pueden visibilizar algunas experiencias educativas exitosas de nuestro país. Las hay en todos los niveles y se dan en el sector público y privado.

Finalmente, una escuela de calidad es aquella que permite empezar a andar la democracia y formar ciudadanos y seres humanos completos, una educación de calidad que permitiría analizar agudamente la idea de Víctor Hugo.

\section{Nota}

1 Subrayado de la autora

\section{Referencias}

Arancibia, V. (1998). "Factores que afectan el rendimiento escolar de los pobres". En E. Cohen (Ed.). Educación, Eficiencia y Equidad. Cap. 7, 157210.Santiago: EPAL/OEA/SUR.

Arancibia, V. (1993). "Aportes y carencias de la enseñanza media en el área cognitiva, afectiva-social, corporal y espiritual: un estudio cualitativo sobre opiniones de jóvenes de sectores populares". Psykhé. 2(1), 81-94.

Arguedas, E. y Bonilla E. (2008). "La participación y su viabilidad en Costa Rica". Revista Educare. XII (Extraordinario), 97-108.

Banco Mundial. (2002) ¿Por qué es el Capital Social importante para el desarrollo? Recuperado de http://web.worldbank.org/ WBSITE/EXTERNAL/TOPICS/ EXTSOCIALDEVELOPMENT/ EXTTSOCIALCAPITAL/0,,content MDK:20187568 menuPK:410388 p agePK:148956 piPK:216618 theSit ePK:401015,00.html

Bellei, C. (2001). “HHa tenido impacto la reforma educativa chilena?" En C. Cox (Ed.), Políticas educacionales en el cambio de siglo. La reforma del sistema escolar de Chile, 125-209. Santiago: Editorial Universitaria. 
Bertucci, J. (2002). "El capital social en los proyectos de alivio de la pobreza". Recuperado de www.cambiocultural. com.ar/investigacion/capitalsocial.htm

Carbajosa, A. (2007). El país de los maestros. Recuperado de http:// elpais.com/diario/2007/02/14/sociedad/1171407607_850215.html

Cassasus, J. y Arancibia, V. (1996). Claves para una educación de calidad. Buenos Aires: Kapelusz.

Guissani, L. (2006). Educar es un riesgo. Madrid: Ediciones Encuentro.

Ednir, M. (2002). "Educación cubana: luces y sombras". Cuadernos Pedagógicos. 308, 71-76.

Espínola, V. (1998). "Revisión de 15 años de política educativa en Chile: ajustes en función de la equidad". En: E. Cohen (Ed.). Educación, Eficiencia y Equidad. Santiago CEPAL/OEA/ SUR.

Estado de la Nación. (2000). Informe del Estado de la Nación de Costa Rica. San José, Costa Rica

Estado de la Educación. (2011). Tercer informe Estado de la Educación. San José, Programa del Estado de la Nación.

Eyzaguirre, B. y Le Foulon, C. (2001). "La calidad de la educación chilena en cifras". Estudio Públicos. 84, 86-204.

Froemel, J. (2002). Conferencia sobre calidad de la educación. Programa LLECE de la UNESCO. Curso: Efectividad y Calidad escolar.

Fullan, M. (2000). El cambio educativo. Buenos Aires: Trillas.

Hoyuelos, A. (s.f.). "Fragmentos de una entrevista realizada por Novedades Educativas”. Reggio Emilia y la pedagogía de Loris Malaguzzi.
Recuperado de www.redsolareargentina.com/notas/Nota_Hoyuelos.pdf

Margery B, E. (2010). Complejidad, transdisciplinariedad y competencias. cinco viñetas pedagógicas. San José: Uruk Editores.

Laboratorio Latinoamericano de la Evaluación de la calidad de la educación. (1998). Primer Informe del Estudio Internacional Comparativo sobre Lenguaje, Matemática y Factores Asociados en Tercero $y$ Cuarto Grado. Recuperado de http:// llece.unesco.cl/medios/pdf/espanol/ Informe-Tec03.pdf.

Laboratorio Latinoamericano de la Evaluación de la calidad de la educación. (2000). Segundo Informe del Estudio Internacional Comparativo sobre Lenguaje, Matemática y Factores Asociados en Tercero $y$ Cuarto Grado. Recuperado de http:// llece.unesco.cl/medios/pdf/espanol/ Informe-Tec03.pdf.

Laboratorio Latinoamericano de la Evaluación de la calidad de la educación. (2002). Estudio cualitativo de escuelas con resultados destacables en siete países latinoamericanos Recuperado de http://unesdoc.unesco. org/images/0013/001310/131037s.pdf

Ramírez, L. (1999). Planificación lingüistica en los programas de estudio de Educación Preescolar: la función de las docentes en la enseñanza de la lengua materna. Tesis de Maestría en Lingüística. Universidad de Costa Rica. Sin publicar.

Stigler, J. y Hiebert, J. (2002). "La brecha de la enseñanza". En Estudios Públicos. 86, 57-144. 